\title{
Rhino-Orbito-Cerebral Mucormycosis in a Diabetic Patient with Idiopathic CD4 + Lymphocytopenia
}

\author{
Nuno Rocha Pereira ${ }^{1}$, Paulo Andrade ${ }^{1}$, Laura Sá ${ }^{1}$, Cândida Abreu ${ }^{1}$, Rita Figueiredo ${ }^{2}$, \\ Roberto Silva ${ }^{3}$, António Sarmento ${ }^{1}$ \\ ${ }^{1}$ Infectious Diseases Department, Centro Hospitalar São João and Faculty of Medicine of Porto University, Porto, Portugal; ${ }^{2}$ Neuro- \\ radiology Department, Centro Hospitalar São João, Porto, Portugal; ${ }^{3}$ Pathology Department, Centro Hospitalar São João and Faculty \\ of Medicine of Porto University, Porto, Portugal. \\ Email:nunopereira85@gmail.com
}

Received September $28^{\text {th }}, 2013$; revised October $26^{\text {th }}, 2013$; accepted November $1^{\text {st }}, 2013$

Copyright (c) 2013 Nuno Rocha Pereira et al. This is an open access article distributed under the Creative Commons Attribution License, which permits unrestricted use, distribution, and reproduction in any medium, provided the original work is properly cited.

\begin{abstract}
A 54-year-old diabetic male, with idiopathic CD4 + lymphocytopenia, was presented with a two-month history of headache and periocular pain followed by vertigo, left hypoacusia and left peripheral facial palsy and hypoesthesia. More than a month after admission, a palate ulcer appeared and Infectious Diseases consultation was required. Mucormycosis was suspected and the diagnosis was confirmed by histologic examination. Despite early surgery and combination antifungal therapy, the patient did not survive. This case report illustrates the difficulty in diagnosing a rare condition with non-specific clinical manifestations and underlines the importance of a timely multidisciplinary approach in order to recognise this highly fatal disease earlier. It also describes a previously non-reported situation of mucormycosis in a patient with idiopathic CD4 + lymphocytopenia.
\end{abstract}

Keywords: Mucormycosis; Idiopathic CD4 + Lymphocytopenia; Diabetes; Amphotericin B

\section{Introduction}

Mucormycosis is a life threatening fungal infection caused by Mucorales fungi. There are six major clinical forms of the disease: cutaneous, pulmonary, gastrointestinal, disseminated, uncommon rare forms (endocarditis, osteomyelitis, peritonitis and renal infection) and rhino-orbito-cerebral (ROCM) [1]. The latter is the most common form and is associated with diabetes in approximately two-thirds of cases [2]. Other risk factors for mucormycosis include haematological malignancies, hematopoietic stem cell and solid organ transplantation, imunossupresssive drugs, deferoxamine therapy and prolonged use of voriconazole [1].

We present a case of ROCM in a diabetic patient with idiopathic CD4 + lymphocytopenia (ICL), to our knowledge, a previously non-described association.

\section{Case Presentation}

An Infectious Diseases consultation was required for a 54-year-old male who had developed an hard palate ulceration. The patient had been admitted 6 weeks before to the Neurology ward with a three-day history of left peripheral facial palsy and left facial hypoesthesia. A brain computed tomography (CT) scan showed a right cerebellar lesion.

His past medical history was remarkable for ICL, diagnosed 5 years earlier after etiologic workup of cryptococcal meningitis; arterial hypertension, obesity, dislipidemia and a poorly controlled type 2 diabetes mellitus were also elicited. For the last five years he was under suppressive therapy with fluconazole and trimethoprim/ sulfamethoxazole plus azithromycin for opportunistic infection prophylaxis.

Two months before admission he started complaining of headache and right periocular pain. One month later, he developed left hypoacusia and vertigo and three days before admission left peripheral facial palsy and hypoesthesia.

On admission at the emergency room (D1) he complained of bilateral frontal headache, bilateral periorbital pain, vertigo, nausea and vomiting. Neurological examination revealed bilateral temporal optic margin blurring, left face hypoesthesia, left peripheral facial palsy, later- 
alized Weber test to the left and positive Romberg sign. A brain CT scan showed a right cerebellar space-occupying lesion with fourth ventricle compression. He was admitted to Neurology ward and brain magnetic resonance imaging (MRI) was performed (D7), revealing a 2 cm right cerebellar nodular lesion with extensive vasogenic oedema which compressed the fourth ventricle and brainstem with right inferior cerebellum amygdala displacement and incipient nonspecific inflammatory process in paranasal sinuses. Dexamethasone was started (D7) due to the early signs of amygdala herniation and risk of hydrocephalus and clinical improvement was observed one week later (D14), with less intense headache and vertigo, while maintaining left face hypoesthesia and left peripheral facial palsy. Extensive workup was performed and occult neoplasia and lymphoproliferative disease were excluded.

After 2 weeks of treatment with dexamethasone (D21), a new MRI was performed, and a significant reduction of the cerebellar lesion's dimension, vasogenic oedema and mass effect was noted. Four days later (D25) the patient started complaining of perinasal pain and epistaxis was observed, followed by worsening periorbital pain (D27) and upper left gingival hypoesthesia (D29). In D32 a rhi- noscopy showed bilateral crests and mucosal hyperemia. In D38 left eye abduction limitation was apparent. Oral examination showed a large darkened hard palate ulcer (Figure 1(a)) and upper teeth loss. At this time a hard palate biopsy was performed and an Infectious Diseases consultation was required. Mucormycosis was suspected and new CT and MRI were performed (D44). MRI revealed pansinusitis, inferior frontal lobe bilateral cerebritis and orbital infiltration (Figure 2). The previously described cerebellar lesion was now not apparent on any of the different MRI sequences. Hard palate biopsy confirmed the clinical suspicion of mucormycosis (Figures 1(b) and (c)) and the patient was started on amphotericin B deoxycholate (1 mg/Kg/day), rapid dexamethasone tapering and aggressive glycaemic control. In the following day (D45) he was submitted to surgery, in which nasal cavity invasion and nasal septum necrosis were observed and necrotic material debridement was performed as well as nasal septum and conchae removal. Successive debridements were made thereafter. Due to renal toxicity, lypossomal amphotericin B was precribed on D49. As the patient was getting worse, combination therapy with caspofungin was initiated three days later (D52), without success. The patient died on D64.

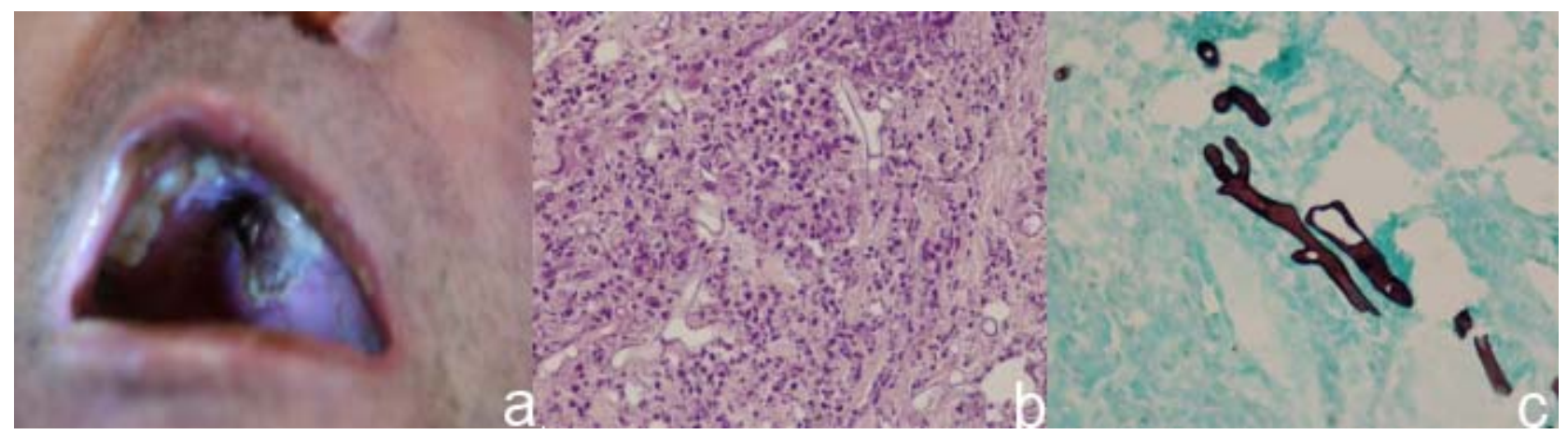

Figure 1. (a) Dark hard palate ulcer; (b) Histopathologic appearance of mucormycosis with large hyphae in a background of necrotic tissue and acute inflammatory process (HE 400×); (c) Grocott methenamine silver staining highlighting those characteristics $(600 \times)$.

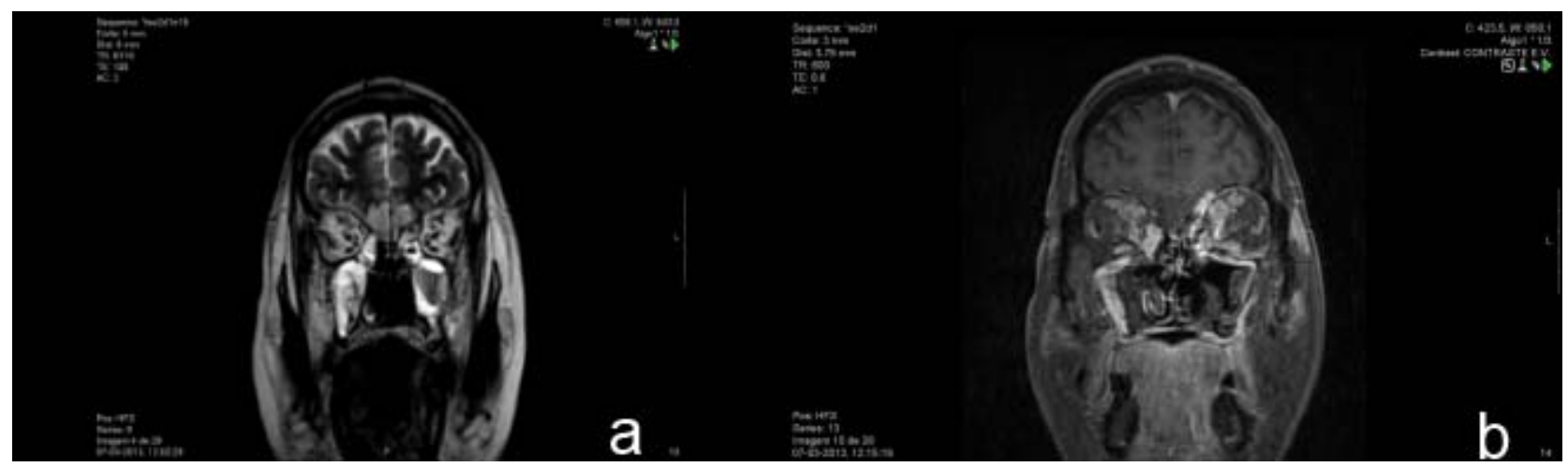

Figure 2. Opacification of paranasal sinus invading extraconic orbital space bilaterally and intracranial extension with inferior frontal encephalitis—cortical hyperintense lesions on T2 (a) along with focal meningeal abnormal enhancement on post gadolinium images (b). 


\section{Discussion}

Mucormycosis is an uncommon infection but its importance has increased in the last years with the growing number of patients with predisposing factors [1]. In Portugal little information about this disease is available, with only three published cases in the literature [3-5], yet the picture is probably similar to other developed countries, where a majority of cases occur in patients with diabetes mellitus, haematological malignancies under chemotherapy and allogeneic stem cell transplant recipients [1].

It is estimated that diabetes is present in 36\% - 88\% of cases and patients with poor glycaemic control and ketoacidosis are the ones at greater risk $[1,6]$. Several mechanisms were proposed to explain the increased risk in diabetic patients and these include qualitative and quantitative abnormalities of neutrophils, monocytes and macrophages and alterations in iron metabolism [2,7]. Chronic corticosteroid therapy also causes defects in macrophages and neutrophils and increases susceptibility to infection by Mucorales $[1,8]$.

The patient in our report had a history of poor glycaemic control despite on-going oral anti-diabetic therapy and although he did not have a history of chronic therapy with corticosteroids, he was put in high-dose dexamethasone. We speculate that corticosteroids may have contributed to the clinical course, by both immunosuppressive effect and worsening glycaemic control.

Prophylactic use of voriconazole in hematopoietic stem cell transplant recipients has increased in recent years and its use probably exerts selective pressure for growth of resistant fungi such as Mucorales [2]. Indeed, voriconazole has been linked with increased incidence of breakthrough mucormycosis in several reports $[9,10]$. Similarly, other agents such as fluconazole, itroconazole and ketoconazole, can produce the same effect [11]. The patient in this case report was taking fluconazole as maintenance therapy for several years and this could have been another contributing factor for developing mucormycosis.

This patient also had a previous diagnosis of ICL, which is characterized by CD4 T-lymphocyte depletion in absence of HIV infection or other immunodeficiency [12]. ICL clinical spectrum ranges from an asymptomatic laboratory abnormality to life-threatening opportunistic conditions similar to those occurring in HIV patients [12]. To our knowledge, mucormycosis is yet to be described in patients with ICL, although several cases were reported in HIV-infected patients [13,14]. Notably, HIV infection in itself does not seem to predispose to Mucormycosis, and in most cases illicit intravenous drug use, neutropenia, diabetes mellitus or corticosteroid therapy are the determinant issues [1]. Given the similar clinical picture of ICL and HIV infection, we consider that ICL may have been an important cofactor for mucormycosis. We also hypothesize that ICL may have acted as a confounder in clinical investigation as the initial diagnostic efforts were directed to conditions more often associated with ICL, further delaying the diagnosis.

The majority of mucormycosis cases present as a subacute illness that might progress over several weeks [8]. Symptoms and signs vary according to clinical form, with ROCM being the most common presentation in diabetic patients [15]. Typically, ROCM is unilateral [8] and the initial signs may be confounded with sinusitis or periorbital cellulitis [1]. Nevertheless, some signs and symptoms, in susceptible individuals, may alert to the diagnosis. These include multiple cranial nerve palsies, unilateral periorbital facial pain, orbital inflammation, eyelid edema, blepharoptosis, proptosis, acute ocular motility changes, internal or external ophtalmoplegia, headache and acute blindness [1]. Our patient initially presented headache, right periocular pain, left peripheral facial palsy and vertigo. Retrospectively, some of these symptoms should have raised concern about mucormycosis but the presence of a cerebellar lesion that could explain some of the symptoms led the medical team to pursue other diagnosis. Also, the unusual occurrence of a bilateral form of ROCM further confounded the differential diagnosis. Other later symptoms and signs were also suggestive of ROCM, such as epistaxis, hard palate painful black necrotic ulceration, teeth loss, gingival hypoesthesia and ophtalmoplegia.

Imaging studies might reveal some indicative signs of ROCM such as sinus mucosal thickening, air-fluid level changes and bone erosion but evidence of infection may be initially absent [8]. Our patient illustrates this situation, having several imaging studies (both brain MRI and CT scans) with incipient signs of sinusitis, though overt clinical symptoms and signs of mucormycosis were already present, emphasizing the importance of considering early surgical exploration with biopsy in high-risk patients [1].

In this case, biopsy was performed late in the course of the disease and the lack of clinical suspicion further delayed the histologic diagnosis that could have been done readily with fresh-frozen section observation [16].

Early diagnosis alongside with reversal of predisposing factors, prompt initiation of antifungal therapy and surgical debridement are crucial for a favorable outcome [15]. Lipid formulations of amphotericin B, particularly liposomal, are associated with better outcomes, probably due to better blood-brain barrier penetration, unique immunomodulatory effects and lesser nephrotoxicity $[2,15]$. Though Mucorales are described as resistant to echinocandins, studies demonstrated that Rhyzopus oryzae, the most common responsible for ROCM, possesses the target enzyme (1,3-beta-D-glucan synthase) for echinocandins, suggesting that these agents might have a therapeutic role in mucormycosis when combined with polyenes 
[2,17]. This theoretical benefit was confirmed in a retrospective study comparing amphotericin B plus caspofungin with amphotericin $\mathrm{B}$ alone and the benefit was particularly evident in patients with cerebral involvement [18]. In spite of the critical role of early anti-fungal therapy, surgery still constitutes the cornerstone for successful treatment of ROCM, since this infection is associated with angioinvasion and extensive necrosis that can impair adequate antifungal therapy to affected tissues [15]. The extent of surgery is dictated by the patient's individual presentation but for maxillofacial infection increasing emphasis is put in less disfiguring surgical procedures [2]. Other therapeutic options for mucormycosis include posaconazole rescue therapy [1], hyperbaric oxygen [19], iron chelation with deferasirox [1] and the use of several kinds of immunotherapy [20].

Our patient was not diagnosed early in the clinical course, yet antifungal therapy was quickly initiated, rapid tapering of corticosteroid and aggressive glycaemic control were performed and surgery was done one day after diagnosis. Due to finantial restrains amphotericin B deoxycolate was the initial therapeutic choice however, once nephrotoxicity was present, liposomal amphotericin $\mathrm{B}$ was started and caspofungin was later added, due to poor therapeutic response. Due to disease extension, with brain and bilateral orbital involvement, a choice was made not to perform aggressive desfigurative surgery and a minimally invasive approach was done.

\section{Conclusion}

This case illustrates the difficulty in diagnosing a rare condition with non-specific clinical manifestations. It also underlines the importance of awareness for risk factors and clinical features of mucormycosis. High index of suspicion, multidisciplinary approach, newer diagnostic and treatment tools altogether should permit, in the future, an improvement in the outcome of this devastating disease.

\section{Acknowledgements}

The authors greet all the professionals involved in management of the patient and thank Dr. Henrique Costa for his article review.

\section{REFERENCES}

[1] G. Petrikkos, A. Skiada, O. Lortholary, E. Roilides, T. J. Walsh and D. P. Kontoyiannis, "Epidemiology and Clinical Manifestations of Mucormycosis," Clinical Infectious Diseases, Vol. 54, No. S1, 2012, pp. S23-S34.

http://dx.doi.org/10.1093/cid/cir866

[2] M. N. Gamaletsou, N. V. Sipsas, E. Roilides and T. J. Walsh, "Rhino-Orbital-Cerebral Mucormycosis," Current Infectious Disease Reports, Vol. 14, No. 4, 2012, pp. 423434. http://dx.doi.org/10.1007/s11908-012-0272-6

[3] J. G. Cortez, A. Speidel, C. Peixoto, E. Selicka, C. Valente, P. Figueiredo and A. Vieira, "Mind the Gap: Management of an Emergent and Threatening Invasive Fungal Infection-A Case Report of Rhino-Orbital-Cerebral and Pulmonary Mucormycosis,” Medical Mycology Case Reports, Vol. 2, 2013, pp. 79-84. http://dx.doi.org/10.1016/j.mmcr.2013.02.008

[4] V. Oliveira and A. Costa, "Cerebral Hematoma Caused by Mucormycosis,” Revista de Neurología, Vol. 33, No. 10, 2001, pp. 951-953.

[5] D. S. Marques, C. Pinho Vaz, R. Branca, et al., "Rhizomucor and Scedosporium Infection Post Hematopoietic Stem-Cell Transplant," Case Reports in Medicine, Vol. 2011, 2011, Article ID: 830769.

[6] M. M. Roden, T. E. Zaoutis, W. L. Buchanan, et al., "Epidemiology and Outcome of Zygomycosis: A Review of 929 Reported Cases,” Clinical Infectious Diseases, Vol. 41, No. 5, 2005, pp. 634-653.

http://dx.doi.org/10.1086/432579

[7] B. Rammaert, F. Lanternier, S. Poiree, R. Kania and O. Lortholary, "Diabetes and Mucormycosis: A Complex Interplay,” Diabetes \& Metabolism, Vol. 38, No. 3, 2012, pp. 193-204.

http://dx.doi.org/10.1016/j.diabet.2012.01.002

[8] U. Wali, A. Balkhair and A. Al-Mujaini, "Cerebro-Rhino Orbital Mucormycosis: An Update,” The Journal of Infection and Public Health, Vol. 5, No. 2, 2012, pp. 116126. http://dx.doi.org/10.1016/j.jiph.2012.01.003

[9] S. M. Trifilio, C. L. Bennett, P. R. Yarnold, et al., "Breakthrough Zygomycosis after Voriconazole Administration among Patients with Hematologic Malignancies Who Receive Hematopoietic Stem-Cell Transplants or Intensive Chemotherapy,” Bone Marrow Transplant, Vol. 39, No. 7, 2007, pp. 425-429.

http://dx.doi.org/10.1038/sj.bmt.1705614

[10] D. P. Kontoyiannis, M. S. Lionakis, R. E. Lewis, et al., "Zygomycosis in a Tertiary-Care Cancer Center in the Era of Aspergillus-Active Antifungal Therapy: A CaseControl Observational Study of 27 Recent Cases,” The Journal of Infectious Diseases, Vol. 191, No. 8, 2005, pp. 1350-1360. http://dx.doi.org/10.1086/428780

[11] L. Pagano, B. Gleissner, L. Fianchi, "Breakthrough Zygomycosis and Voriconazole," The Journal of Infectious Diseases, Vol. 192, No. 8, 2005, pp. 1496-1497. http://dx.doi.org/10.1086/444433

[12] L. Luo and T. Li, "Idiopathic CD4 Lymphocytopenia and Opportunistic Infection-An Update,” FEMS Immunology and Medical Microbiology, Vol. 54, No. 3, 2008, pp. 283-289.

http://dx.doi.org/10.1111/j.1574-695X.2008.00490.x

[13] S. Antinori, M. Nebuloni, C. Magni, et al., "Trends in the Postmortem Diagnosis of Opportunistic Invasive Fungal Infections in Patients with AIDS: A Retrospective Study of 1630 Autopsies Performed between 1984 and 2002,” American Journal of Clinical Pathology, Vol. 132, No. 2, 2009, pp. 221-227.

http://dx.doi.org/10.1309/AJCPRAAE8LZ7DTNE

[14] L. Nichols, R. Z. Ocque and I. Daly, “Zygomycosis Asso- 
ciated with HIV Infection and Liver Transplantation,” Pathology Research International, Vol. 2011, 2011, Article ID: 545981.

[15] H. Y. Sun and N. Singh, "Mucormycosis: Its Contemporary Face and Management Strategies," The Lancet Infectious Diseases, Vol. 11, No. 4, 2011, pp. 301-311. http://dx.doi.org/10.1016/S1473-3099(10)70316-9

[16] T. J. Walsh, M. N. Gamaletsou, M. R. McGinnis, R. T. Hayden and D. P. Kontoyiannis, "Early Clinical and Laboratory Diagnosis of Invasive Pulmonary, Extrapulmonary, and Disseminated Mucormycosis (Zygomycosis)," Clinical Infectious Diseases, Vol. 54, No. S1, 2012, pp. S55-S60. http://dx.doi.org/10.1093/cid/cir868

[17] B. Spellberg, A. Ibrahim, E. Roilides, et al., "Combination Therapy for Mucormycosis: Why, What, and How?" Clinical Infectious Diseases, Vol. 54, No. S1, 2012, pp.
S73-S78. http://dx.doi.org/10.1093/cid/cir885

[18] C. Reed, R. Bryant, A. S. Ibrahim, et al., "Combination Polyene-Caspofungin Treatment of Rhino-Orbital-Cerebral Mucormycosis," Clinical Infectious Diseases, Vol. 47, No. 3, 2008, pp. 364-371. http://dx.doi.org/10.1086/589857

[19] A. Tragiannidis and A. H. Groll, "Hyperbaric Oxygen Therapy and Other Adjunctive Treatments for Zygomycosis," Clinical Microbiology and Infection, Vol. 15, No. S5, 2009, pp. 82-86.

http://dx.doi.org/10.1111/j.1469-0691.2009.02986.x

[20] A. Safdar, "Immunotherapy for Invasive Mold Disease in Severely Immunosuppressed Patients,” Clinical Infectious Diseases, Vol. 57, No. 1, 2013, pp. 94-100. http://dx.doi.org/10.1093/cid/cit187 